\title{
CIRCLES IN COMPACT HOMOGENEOUS RIEMANNIAN SPACES AND IMMERSIONS OF FINITE TYPE*
}

\author{
BANG-YEN CHEN \\ Department of Mathematics, Michigan State University, East Lansing, MI 48824-1027, U.S.A. \\ e-mail:bychen@math.msu.edu
}

(Received 25 April, 2000; revised 6 November, 2000)

\begin{abstract}
A unit speed curve $\gamma=\gamma(s)$ in a Riemannian manifold $N$ is called a circle if there exists a unit vector field $Y(s)$ along $\gamma$ and a positive constant $k$ such that $\nabla_{s} \gamma^{\prime}(s)=k Y(s), \nabla_{s} Y(s)=-k \gamma^{\prime}(s)$. The main purpose of this article is to investigate the fundamental relationships between circles, maximal tori in compact symmetric spaces, and immersions of finite type.
\end{abstract}

2000 Mathematics Subject Classification. Primary 53C35, 53C40. Secondary $53 \mathrm{C} 30$.

1. Introduction. A unit speed curve $\gamma=\gamma(s)$ in a Riemannian manifold $N$ is called a circle if there exists a vector field $Y(s)$ of unit vectors along $\gamma$ and a positive constant $k$ such that $\nabla_{s} X(s)=k Y(s), \nabla_{s} Y(s)=-k X(s)$, where $X(s)$ denotes the unit tangent vector of $\gamma$ and $\nabla_{s}$ the covariant differentiation along $\gamma$ at each point $\gamma(s)$. The number $1 / k$ is called the radius of the circle $\gamma$.

Let $M$ be a Riemannian manifold. All isometries $I(M)$ of $M$ form a Lie group. Let $G_{M}$ be the connected component of $I(M)$ that is compact if $M$ is compact. A Riemannian manifold $M$ is called homogeneous if $G_{M}$ acts transitively on $M$. Denote by $K_{M}$ the isotropy subgroup at a point $o$ in $M$. We have $M=G_{M} / K_{M}$. Very often, we simply denote $G_{M}$ and $K_{M}$ by $G$ and $K$, respectively.

A curve $\gamma$ in $M=G_{M} / K_{M}$ is called a homogenous curve if it is the orbit of a point under the action of a one-parameter subgroup $\left\{\phi_{t}\right\}$ of $G_{M}$. The linear isotropy representation of $M$ is the orthogonal representation of $K$ over the tangent space $T_{o} M$ at $o$ defined by $K \rightarrow O\left(T_{o} M\right): \phi \mapsto\left(\phi_{*}\right)_{o}$, where $\left(\phi_{*}\right)_{o}$ denotes the differential of the isometry $\phi$ at $o$. A homogeneous Riemannian manifold is said to be isotropyirreducible if its linear isotropy representation is irreducible. Let $\langle$,$\rangle denote the$ $\operatorname{Ad}_{G}(K)$-invariant inner product on $M=G / K$. Let $g$ and $\mathfrak{f}$ be the Lie algebras of $G$ and $K$, and $\mathfrak{g}=\mathfrak{f} \oplus \mathfrak{m}$ the Cartan decomposition of $\mathfrak{g}$. $M$ is called naturally reductive if $\left\langle[Z, X]_{\mathfrak{m}}, Y\right\rangle+\left\langle X,[Z, Y]_{\mathfrak{m}}\right\rangle=0$, for $X, Y, Z \in \mathfrak{m}$, where $[,]_{\mathfrak{m}}$ is the $\mathfrak{m}$-component of the Lie bracket in $g$.

The purpose of this article is to investigate the fundamental relationships between circles, maximal tori in compact symmetric spaces, and immersions of finite type. Our main results are the following.

(a) An isometric immersion of a compact irreducible symmetric space $M$ into Euclidean space is of finite type if and only if it carries maximal tori of $M$ into submanifolds of finite type.

*Dedicated to Professor Koichi Ogiue on the occasion of his sixtieth birthday. 
(b) If an isometric immersion of a compact rank one symmetric space carries circles into curves of finite type, then it is of finite type.

(c) If $x: M \rightarrow \mathbb{E}^{m}$ is a finite type isometric immersion of a compact irreducible symmetric space, then $M$ is of rank one if and only if, for some $r>0, x$ carries circles with radius $r$ into curves of finite type.

(d) Every finite type isometric immersion of a compact irreducible homogeneous space carries homogeneous curves into curves of finite type.

(e) A finite type isometric immersion of a (flat) torus carries every circle of the torus into a curve of infinite type.

2. Submanifolds and curves of finite type. In this section we review briefly some basic facts on submanifolds of finite type. (For the details, see $[\mathbf{1 , 3}, \mathbf{4 , 1 2}$.) Let $M$ be a compact Riemannian manifold and let $f$ be a nonconstant function in $C^{\infty}(M)$. Then one can make the following spectral resolution (or decomposition): $f=f_{0}+\sum_{i=1}^{\infty} f_{i}$, $\Delta f_{i}=\lambda_{i} f_{i}$. The set $T(f)=\left\{i>0: f_{i} \neq 0\right\}$ is called the type of $f$. The function $f$ is said to be of finite type if $T(f)$ is a finite set; and $f$ is of infinite type if $T(f)$ is an infinite set. Moreover, $f$ is said to be of $k$-type if $T(f)$ contains exactly $k$ elements. The upper order u.o. $(f)$ and the lower order 1.o. $(f)$ of $f$ are defined respectively by u.o. $(f)=$ sup $T(f)$ and 1.o. $(f)=\min T(f)$. The same can be repeated for vector-valued differentiable functions on $M$. In particular, we can define the notions of type, order, finite type, infinite type, $k$-type, upper order and lower order for isometric immersions of a compact Riemannian manifold into Euclidean space.

Let $\gamma: S^{1} \rightarrow \mathbb{E}^{m}$ be an isometric immersion of a closed smooth curve of length $2 \pi r$ into $\mathbb{E}^{m}$. Denote by $s$ the arc length of $S^{1}$. For a periodic function $f=f(s)$ with period $2 \pi r, f(s)$ has a Fourier series expansion given by

$$
f(s)=\frac{a_{0}}{2}+\sum_{j=1}^{\infty}\left(a_{j} \cos \left(\frac{j s}{r}\right)+b_{j} \sin \left(\frac{j s}{r}\right)\right),
$$

where $a_{0}$ is a constant and $a_{j}, b_{j}$ are the Fourier coefficients.

In terms of Fourier series expansion, we have the following result. See [3].

Proposition 2.1. Let $\gamma: S^{1} \rightarrow \mathbb{E}^{m}$ be a closed smooth curve of length $2 \pi r$ in $\mathbb{E}^{m}$. Then $\gamma$ is of finite type if and only if the Fourier series expansion of each coordinate function of $\gamma$ has only finite nonzero terms.

Proposition 2.1 can be restated as follows.

Proposition 2.2. Let $\gamma: S^{1} \rightarrow \mathbb{E}^{m}$ be a closed smooth curve of length $2 \pi r$ in $\mathbb{E}^{m}$. Then $\gamma$ is of finite type if and only if each coordinate function of $\gamma$ is an algebraic polynomial in $\cos s$ and $\sin s$.

Proof. This follows from Proposition 2.1, the facts that $\cos (n s)$ and $\sin (n s)$ are algebraic polynomials of degree $n$ in $\cos s$ and $\sin s$, and every algebraic polynomial of degree $n$ in $\cos s$ and $\sin s$ can be expressed as a function of finite type.

Let $T^{n}$ denote the (flat) torus $\mathbb{E}^{n} / \Lambda_{T^{n}}$, where $\Lambda_{T^{n}}$ is a lattice of $\mathbb{E}^{n}$. The dual lattice of $\Lambda_{T^{n}}$ is given by $\Lambda_{T^{n}}^{*}=\left\{u \in \mathbb{E}^{n}:\langle u, v\rangle \in \mathbb{Z}\right.$ for any $\left.v \in \Lambda_{T^{n}}\right\}$. For each 
$x \in \Lambda_{T^{n}}^{*}$, we define $f_{x}$ on $\mathbb{E}^{n}$ by $f_{x}(y)=e^{2 \pi i x(y)}$, where $y \in \mathbb{E}^{n}$ on the right is regarded as a vector. Then $f_{x}$ defines a function on $T^{n}$, also denote by $f_{x}$, satisfying $\Delta f_{x}=4 \pi^{2}\|x\|^{2} f_{x}$. Thus, $u_{x}(y)=\cos (2 \pi x(y))$ and $v_{x}(y)=\sin (2 \pi x(y))$ are eigenfunctions of the Laplacian with eigenvalue $\lambda=4 \pi^{2}\|x\|^{2}$ for each $x \in \Lambda_{T^{n}}^{*}$. By an $r$-dimensional subtorus $T^{r}(r \geq 2)$ of an $n$-torus $T^{n}$, we mean a compact totally geodesic submanifold of dimension $r$ in $T^{n}$.

We need the following lemma.

Lemma 2.3. Let $\varphi: T^{n} \rightarrow \mathbb{E}^{m}$ be a finite type isometric immersion of a $T^{n}$ into $\mathbb{E}^{m}$. Then $\varphi$ maps every subtorus $T^{r}$ of $T^{n}$ into a submanifold of finite type.

Proof. It suffices to prove that the restriction to a subtorus $T^{r}$ of an eigenfunction of the Laplacian $\Delta^{T^{n}}$ is an eigenfunction of $\Delta^{T^{r}}$. However, this is clear because any linear map $\mathbb{E}^{n} \rightarrow \mathbf{R}$ which takes integer values on $\Lambda_{T^{n}}$ also takes integer values on the sublattice $\Lambda_{T^{r}} \subset \Lambda_{T^{n}}$.

3. Homogeneous curves of finite type. If $M$ is a naturally reductive homogeneous Riemannian manifold, then every geodesic of $M$ is homogeneous [11, p. 313]. On the other hand, not every circle in a naturally reductive homogeneous Riemannian manifold is homogeneous. In fact, every circle of a homogeneous Riemannian manifold $M$ is homogeneous if and only if $M$ is either a Euclidean space or a symmetric space of rank one [9].

Let $x: M=G / K \rightarrow \mathbb{E}^{m}$ be a $G$-equivariant isometric immersion of a compact Riemannian homogeneous manifold. Then there is a Lie homomorphism $\sigma$ of $G$ into $I\left(\mathbb{E}^{m}\right)$ such that $x(g(p))=\sigma(g)(x(p))$, for every $g \in G$ and $p \in M$.

Lemma 3.1. Let $x: M=G / K \rightarrow \mathbb{E}^{m}$ be a $G$-equivariant isometric immersion of a compact homogeneous Riemannian manifold $M=G / K$ into $\mathbb{E}^{m}$. Then $x$ carries every homogeneous curve in $M$ into a curve of finite type. In particular, $x$ carries every geodesic in a compact naturally reductive homogeneous Riemannian manifold into a curve of finite type.

Proof. Let $\gamma$ be a homogeneous curve in $M=G / K$ and let $x: M \rightarrow \mathbb{E}^{m}$ be a $G$-equivariant isometric immersion. Then $x(\gamma)$ is homogeneous. Thus $\Delta_{\gamma} H_{\gamma}, \Delta_{\gamma}^{2} H_{\gamma}$, $\ldots, \Delta_{\gamma}^{m+1} H_{\gamma}$ are linearly dependent, where $\Delta_{\gamma}=-d^{2} / d s^{2}$ and $H_{\gamma}=d^{2} \gamma / d s^{2}$ is the mean curvature vector of $\gamma$. Hence, there is a nontrivial polynomial $P$ of degree $m+1$ such that $P\left(\Delta_{\gamma}\right) H_{\gamma}=0$. Therefore $x(\gamma)$ is a curve of finite type [6].

The second statement follows from the fact that geodesics in a natural reductive homogeneous Riemannian manifold are homogeneous.

For each eigenvalue $\lambda$ of the Laplacian on a compact irreducible homogeneous space $M$, let $\phi_{1}, \ldots, \phi_{m_{\lambda}}$ be an orthonormal basis of the eigenspace with eigenvalue $\lambda$, where $m_{\lambda}$ is the multiplicity of $\lambda$. We define a map $\psi_{\lambda}: M \rightarrow \mathbb{E}^{m_{\lambda}}$ by $\psi_{\lambda}(u)=\left(c_{\lambda} / m_{\lambda}^{2}\right)\left(\phi_{1}(u), \ldots, \phi_{m_{\lambda}}(u)\right)$, where $c_{\lambda}$ is a positive number. For a suitable constant $c_{\lambda}>0$, the map $\psi_{\lambda}$ defines an isometric minimal immersion of $M$ into the unit hypersphere $S_{0}^{m_{\lambda}-1}(1)$ centered at the origin of $\mathbb{E}^{m_{\lambda}}$. Thus, by a result of Takahashi, each such $\psi_{\lambda}$ is a 1-type isometric immersion. If $\lambda_{i}$ is the $i$-th nonzero 
eigenvalue of the Laplacian of $M$, then $\psi_{i}=\psi_{\lambda_{i}}$ is called the $i$-th standard immersion of $M$. It is known that each standard immersion of $M=G / K$ is $G$-equivariant.

Let $\psi_{t_{i}}: M \rightarrow \mathbb{E}^{m_{i}}, i=1, \ldots, k$, be $k$ standard isometric immersions of a compact irreducible homogeneous Riemannian manifold $M$. Suppose $a_{t_{1}}, \ldots, a_{t_{k}}$ are constants satisfying $a_{t_{1}}^{2}+\cdots+a_{t_{k}}^{2}=1$. Let $D\left(a_{t_{1}}, \ldots, a_{t_{k}}\right)$ denote the immersion of $M$ into $\mathbb{E}^{N}, N=m_{1}+\cdots+m_{k}$, defined by $D\left(a_{t_{1}}, \ldots, a_{t_{k}}\right)=\left(a_{t_{1}} \psi_{t_{1}}, \ldots, a_{t_{k}} \psi_{t_{k}}\right)$. Then $D\left(a_{t_{1}}, \ldots, a_{t_{k}}\right)$ is an isometric immersion which is called a standard diagonal immersion. Standard diagonal immersions of $M=G / K$ are $G$-equivariant, too.

Theorem 3.2. Let $x: M \rightarrow \mathbb{E}^{m}$ be a finite type isometric immersion of a compact irreducible homogeneous Riemannian manifold $M$. Then $x$ carries every homogeneous curve in $M$ into a curve of finite type.

Proof. Let $x: M \rightarrow \mathbb{E}^{m}$ be a finite type isometric immersion of a compact irreducible homogeneous Riemannian manifold $M$ into $\mathbb{E}^{m}$. Without loss of generality we may assume that the center of mass of $x$ in $\mathbb{E}^{m}$ is chosen to be the origin of $\mathbb{E}^{m}$. Assume that the type of $x$ is given by $T(x)=\left\{t_{1}, t_{2}, \ldots, t_{k}\right\}$.

Let $\left\{\phi_{1}^{t_{i}}, \ldots, \phi_{m_{t_{i}}}^{t_{i}}\right\}$ be an orthonormal basis of the eigenspace $V_{t_{i}}$ with eigenvalue $\lambda_{t_{i}}$. Then the position vector field of $M$ in $\mathbb{E}^{m}$ takes the form

$$
x=\sum_{i=1}^{k}\left(a_{1}^{i} \phi_{1}^{t_{i}}+\cdots+a_{m_{t_{i}}}^{i} \phi_{m_{t_{i}}}^{t_{i}}\right),
$$

where $a_{1}^{1}, \ldots, a_{m_{t_{1}}}^{1}, \ldots, a_{1}^{k}, \ldots, a_{m_{t_{k}}}^{k}$ are vectors in $\mathbb{E}^{m}$. Consider a standard diagonal isometric immersion $D\left(c_{t_{1}}, \ldots, c_{t_{k}}\right)$ of $M$ whose type is the same as the type of $x$. Then $D=D\left(c_{t_{1}}, \ldots, c_{t_{k}}\right)$ takes the form

$$
D=\left(c_{t_{1}} b_{t_{1}} \phi_{1}^{t_{1}}, \ldots, c_{t_{1}} b_{t_{1}} \phi_{m_{t_{1}}}^{t_{1}}, \ldots, c_{t_{k}} b_{t_{k}} \phi_{1}^{t_{k}}, \ldots, c_{t_{k}} b_{t_{k}} \phi_{m_{t_{k}}}^{t_{k_{k}}}\right)
$$

for some positive numbers $b_{t_{1}}, \ldots, b_{t_{k}}$. Choose a standard basis $\left\{\epsilon_{1}, \ldots, \epsilon_{N}\right\}$ of $\mathbb{E}^{N}$. Consider the linear map $L: \mathbb{E}^{N} \rightarrow \mathbb{E}^{m}$ defined by

$$
\begin{aligned}
& L\left(c_{t_{1}} b_{t_{1}} \epsilon_{1}\right)=a_{1}^{1}, \ldots, L\left(c_{t_{1}} b_{t_{1}} \epsilon_{m_{t_{1}}}\right)=a_{m_{t_{1}}}^{1}, \ldots, \\
& L\left(c_{t_{\ell}} b_{t_{k}} \epsilon_{m_{t_{1}}+\cdots+m_{t_{k-1}}+1}\right)=a_{1}^{k}, \ldots, L\left(c_{t_{k}} b_{t_{k}} \epsilon_{m_{t_{1}}+\cdots+m_{t_{k}}}\right)=a_{m_{t_{k}}}^{k}, \\
& L\left(\epsilon_{m_{t_{1}}+\cdots+m_{t_{k}}+1}\right)=\cdots=L\left(\epsilon_{N}\right)=0 .
\end{aligned}
$$

Then $x=L \circ D\left(c_{t_{1}}, \ldots, c_{t_{k}}\right)$. Since a standard diagonal immersion of $M$ is $G$-equivariant, $D\left(c_{t_{1}}, \ldots, c_{t_{k}}\right)$ is $G$-equivariant. Thus the restriction of $D\left(c_{t_{1}}, \ldots, c_{t_{k}}\right)$ to each homogeneous curve $\gamma$ of $M$ is equivariant. Hence, by Lemma 3.1, the diagonal immersion $D\left(c_{t_{1}}, \ldots, c_{t_{k}}\right)$ maps every homogeneous curve in $M$ into a curve of finite type. Thus, the Fourier series expansion of $D\left(c_{t_{1}}, \ldots, c_{t_{k}}\right)(\gamma)$ must consist of only finitely many nonzero terms, by Proposition 2.1. Because $x$ is the composition of $D\left(c_{t_{1}}, \ldots, c_{t_{k}}\right)$ followed by a linear map $L$, the Fourier series expansion of $x(\gamma)$ must consist of only finitely many nonzero terms, too. Therefore, $x$ must carry homogeneous curves in $M$ into curves of finite type. 
4. Maximal flat tori and finite type immersions. We need the following lemma from $[4,7]$.

Lemma 4.1. Let $x: M \rightarrow \mathbb{E}^{m}$ be an isometric immersion of a compact irreducible symmetric space. Then $x$ is of finite type if and only if $x$ carries each geodesic of $M$ into a curve of finite type.

A maximal flat torus of a compact symmetric space is a complete flat totally geodesic submanifold of maximal possible dimension [8]. The following result is a natural extension of Lemma 4.1.

Theorem 4.2. (1) Let $x: M \rightarrow \mathbb{E}^{m}$ be a finite type isometric immersion of a compact irreducible symmetric space. Then $x$ maps every complete irreducible totally geodesic submanifold of $M$ into a submanifold of finite type.

(2) An isometric immersion $x: M \rightarrow \mathbb{E}^{m}$ of a compact irreducible symmetric space is of finite type if and only if it carries every maximal flat torus $T^{r}$ of $M$ into a submanifold of finite type.

Proof. (1) Let $B$ be a complete irreducible totally geodesic submanifold of a compact irreducible symmetric space $M$. If $\operatorname{dim} B=1$, the result follows from Lemma 4.1. If $\operatorname{dim} B \geq 2$, then $B$ is also a compact irreducible symmetric space. Since $x: M \rightarrow \mathbb{E}^{m}$ is of finite type and geodesics of $B$ are geodesics of $M, x$ carries each geodesic of $B$ into a curve of finite type. Therefore, the restriction of $x$ to $B$ is an isometric immersion of finite type by Lemma 4.1.

(2) Assume $x: M \rightarrow \mathbb{E}^{m}$ is an isometric immersion of a compact irreducible symmetric space that carries every maximal flat torus into a submanifold of finite type. Suppose $\gamma=\gamma(s)$ is a geodesic in $M$. Then $\gamma$ is contained in a maximal flat torus of $M$, say $T^{r}$, where $r=\operatorname{rank}(M)$. Assume $T^{r}=\mathbb{E}^{r} / \Lambda$ for some lattice $\Lambda$. Since the restriction of $x$ to $T^{r}$ is of finite type, there is a finite subset $F=\left\{\mu_{1}, \ldots, \mu_{k}\right\}$ of $\operatorname{Spec}\left(T^{r}\right)$ such that each coordinate function of $\left.x\right|_{T^{r}}: T^{r} \rightarrow \mathbb{E}^{m}$ is a linear combination of the eigenfunctions associated with the eigenvalues $\left\{\mu_{1}, \ldots, \mu_{k}\right\}$. Put

$$
S=\left\{x \in \Lambda^{*}:\|x\|^{2}=\frac{\mu_{j}}{4 \pi^{2}} \text { for some } j=1, \ldots, k\right\}
$$

and let $N$ be the cardinal number $\# S$. Assume $S=\left\{\left(a_{1}^{i}, \ldots, a_{n}^{i}\right), i=1, \ldots, N\right\}$. Then each coordinate function $\varphi_{A}$ of $\varphi: T^{r} \rightarrow \mathbb{E}^{m}$ takes the following form:

$$
\varphi_{A}=\sum_{i=1}^{N}\left[b_{A i} \cos \left(2 \pi \sum_{j=1}^{r} a_{j}^{i} y_{j}\right)+c_{A i} \sin \left(2 \pi \sum_{j=1}^{r} a_{j}^{i} y_{j}\right)\right], \quad A=1, \ldots, m,
$$

for some constants $b_{A i}, c_{A i}$. Without loss of generality, we may assume that the geodesic $\gamma$ is defined by $y_{j}(s)=\gamma_{j} s, \gamma_{j} \in \mathbf{R}$, for $j=1, \ldots, r$. Then we obtain $\varphi_{A}=\sum_{i=1}^{N}\left[b_{A i} \cos \left(2 \pi r_{i} s\right)+c_{A i} \sin \left(2 \pi r_{i} s\right)\right], r_{i}=\sum_{j=1}^{r} \gamma_{j} a_{j}^{i}$ which implies that $\gamma(s)$ is of finite type. Since $\gamma$ is an arbitrary geodesic in $M, x: M \rightarrow \mathbb{E}^{m}$ is of finite type, by Lemma 4.1.

Conversely, assume $x$ is a finite type isometric immersion of a compact irreducible symmetric space $M$. If $\operatorname{rank}(M)=1$, then Lemma 4.1 implies that $x$ carries 
maximal flat tori into submanifolds of finite type. If $\operatorname{rank}(M) \geq 2$, Lemma 4.1 implies that $x$ carries geodesics of $T^{r}$ into curves of finite type. In order to prove that $T^{r}$ is a submanifold of finite type via $x$, it suffices to prove that every infinite type isometric immersion of $T^{r}$ carries some geodesics in $T^{r}$ into curves of infinite type. This can be done as follows.

Assume $T^{r}$ is isometric to $\mathbb{E}^{r} / \Lambda$ for some lattice $\Lambda$ in $\mathbb{E}^{r}$. If $\psi: T^{r} \rightarrow \mathbb{E}^{m}$ is an infinite type isometric immersion, then there is a coordinate function of $\psi$, say $\psi_{1}$ whose upper order is $\infty$. Assume $\psi_{1}$ takes the form:

$$
\psi_{1}=\sum_{a \in \Lambda^{*}}\left[b_{a} \cos \left(2 \pi \sum_{j=1}^{r} a_{j} y_{j}\right)+c_{a} \sin \left(2 \pi \sum_{j=1}^{r} a_{j} y_{j}\right)\right],
$$

where $a=\left(a_{1}, \ldots, a_{r}\right), a \in \Lambda^{*}$, and $b_{a}, c_{a}$ are constants. Since the upper order of $\psi_{1}$ is $\infty$, there is a subset $W$ of $\Lambda^{*}$ such that $b_{a}, c_{a}$ are not both zero for each $a \in W$ and $W$ satisfies $\sup _{a \in W}\|a\|=\infty$. Hence, there exists an $i \in\{1, \ldots, r\}$ such that $\sup _{a_{i}^{2} \in W}\left(a_{i}\right)^{2}=\infty$. Without loss of generality, we may assume $i=1$. Consider the geodesic in $T^{r}$ which is induced from the unit speed curve $\gamma(s)=(s, 0, \ldots, 0)$ in $\mathbb{E}^{r}$ via $\pi_{\Lambda}: \mathbb{E}^{r} \rightarrow T^{r}$. The first coordinate function of the curve $\psi(\gamma)$ is then given by $(\psi \circ \gamma)_{1}=\sum_{a \in \Lambda^{*}}\left[b_{a} \cos \left(2 \pi a_{1} s\right)+c_{a} \sin \left(2 \pi a_{1} s\right)\right]$. Since $\sup _{a \in W}\left(a_{1}\right)^{2}=\infty$ and $b_{a}, c_{a}$ are not both zero, for any $a \in W$, the curve $\psi \circ \gamma$ is thus of infinite type.

5. Circles in homogeneous spaces. Let $T^{2}=\mathbb{E}^{2} / \Lambda$ be a 2-torus, where $\Lambda$ is generated by $(2 \pi, 0)$ and $(0,2 \pi)$. Then $T^{2}$ is isometric to the product of two circles. Let $\pi_{\Lambda}: \mathbb{E}^{2} \rightarrow T^{2}$ be the projection. Consider the isometric immersion of $\mathbb{E}^{2}$ into $\mathbb{E}^{4}$ defined by $\psi: \mathbb{E}^{2} \ni(u, v) \mapsto(\cos u, \sin u, \cos v, \sin v) \in \mathbb{E}^{4}$. Then $\psi$ induces an isometric imbedding $\varphi: T^{2} \rightarrow \mathbb{E}^{4}$. If $\gamma$ is the circle of radius $r$ defined by $\gamma(s)=$ $(r \cos (s / r), r \sin (s / r))$, then $\tilde{\gamma}=\pi_{\Lambda}(\gamma)$ is a circle of radius $r$ in $T^{2}$. Clearly, $\varphi$ carries $\tilde{\gamma}$ into the following curve in $\mathbb{E}^{4}$ :

$$
\varphi \circ \tilde{\gamma}=\left(\cos \left(r \cos \left(\frac{S}{r}\right)\right), \sin \left(r \cos \left(\frac{S}{r}\right)\right), \cos \left(r \sin \left(\frac{S}{r}\right)\right), \sin \left(r \sin \left(\frac{S}{r}\right)\right)\right) .
$$

Obviously,

$$
\cos \left(r \cos \left(\frac{S}{r}\right)\right)=1-\frac{r^{2}}{2 !} \cos ^{2}\left(\frac{S}{r}\right)+\frac{r^{4}}{4 !} \cos ^{4}\left(\frac{S}{r}\right)-\frac{r^{6}}{6 !} \cos ^{6}\left(\frac{S}{r}\right)+\cdots
$$

is an infinite series which is not an algebraic polynomial in $\cos (s / r)$ and $\sin (s / r)$. Hence, $\cos (r(\cos (s / r))$ is of infinite type by Proposition 2.2. Thus, $\varphi \circ \tilde{\gamma}$ is of infinite type.

REMARK 5.1. Another way to see that $\cos (r \cos (s / r))$ is of infinite type is to apply the theory of holomorphic functions. This can be done as follows.

Let $z=\exp (i s / r)$. Then $2 \cos \left(r \cos \left(\frac{S}{r}\right)\right)=e^{i r\left(z+z^{-1}\right) / 2}+e^{-i r\left(z+z^{-1}\right) / 2}$ is a holomorphic function which has two singular points $z=0, \infty$. Obviously these are essentially singular points. But trigonometric polynomials are precisely those analytic functions having $0, \infty$ as singular points for which these points are not essentially singular points. Thus, $\cos (r \cos (s / r))$ must be of infinite type. 
For a general finite type torus, we have the following result.

TheORem 5.1. Let $\varphi: T^{n} \rightarrow \mathbb{E}^{m}$ be a finite type isometric immersion of an $n$-torus $T^{n}$. Then $\varphi$ carries each circle in $T^{n}$ into a curve of infinite type.

Proof. Since every circle $\gamma$ in $T^{n}$ is contained in a 2-dimensional subtorus $T^{2}$ of $T^{n}$, we only need to prove the result for $n=2$ according to Lemma 2.3 .

Let $\varphi: T^{2} \rightarrow \mathbb{E}^{m}$ be a finite type isometric immersion. Then there is a finite subset $F=\left\{\mu_{1}, \ldots, \mu_{k}\right\} \subset \operatorname{Spec}\left(T^{2}\right)$, so that each coordinate function of $\varphi$ is a linear combination of eigenfunctions associated with the eigenvalues $\left\{\mu_{1}, \ldots, \mu_{k}\right\}$.

Put $S=\left\{x \in \Lambda^{*}:\|x\|^{2}=\mu_{j} / 4 \pi^{2}\right.$ for some $\left.j=1, \ldots, k\right\}$. Then $S$ is a finite set because the multiplicity of each eigenvalue in $\left\{\mu_{1}, \ldots, \mu_{k}\right\}$ is finite. Denote the cardinal number $\# S$ by $N$. Assume $S=\left\{\left(a_{i}, b_{i}\right), i=1, \ldots, N\right\}$. Then each coordinate function $\varphi_{A}(A=1, \ldots, m)$ of $\varphi: T^{2} \rightarrow \mathbb{E}^{m}$ takes the following form:

$$
\varphi_{A}\left(y_{1}, y_{2}\right)=\sum_{i=1}^{N}\left[c_{A i} \cos \left(2 \pi\left(a_{i} y_{1}+b_{i} y_{2}\right)\right)+d_{A i} \sin \left(2 \pi\left(a_{i} y_{1}+b_{i} y_{2}\right)\right)\right]
$$

for some constants $c_{A i}, d_{A i}$. Let $\gamma=\gamma(s)$ be a circle with radius $r$ in $T^{2}$. Without loss of generality, we may assume $\gamma$ is obtained from the circle $(r \cos (s / r), r \sin (s / r))$ via $\pi_{\Lambda}: \mathbb{E}^{2} \rightarrow T^{2}$. The $A$-th coordinate function of $\varphi(\gamma)$ in $\mathbb{E}^{m}$ is then given by

$$
\begin{aligned}
(\varphi \circ \gamma)_{A}=\sum_{i=1}^{N} & \left\{c_{A i} \cos \left(2 \pi r\left(a_{i} \cos \left(\frac{S}{r}\right)+b_{i} \sin \left(\frac{S}{r}\right)\right)\right)\right. \\
& \left.+d_{A i} \sin \left(2 \pi r\left(a_{i} \cos \left(\frac{S}{r}\right)+b_{i} \sin \left(\frac{S}{r}\right)\right)\right)\right\} .
\end{aligned}
$$

Since $\varphi$ is isometric, there is at least one coordinate function, say $(\varphi \circ \gamma)_{1}$, that is not a constant. Thus, $(\varphi \circ \gamma)_{1}$ takes the form

$$
\begin{aligned}
(\varphi \circ \gamma)_{1}=\sum_{i=1}^{N} & \left\{c_{i} \cos \left(2 \pi r\left(\alpha_{i} \cos \left(\frac{S}{r}\right)+\beta_{i} \sin \left(\frac{S}{r}\right)\right)\right)\right. \\
+ & \left.d_{i} \sin \left(2 \pi r\left(\alpha_{i} \cos \left(\frac{S}{r}\right)+\beta_{i} \sin \left(\frac{S}{r}\right)\right)\right)\right\},
\end{aligned}
$$

where $c_{1}, \ldots, c_{N}, d_{1}, \ldots, d_{N}$ are constants, not all zero, and $\alpha_{1}, \ldots, \alpha_{n}, \beta_{1}, \ldots, \beta_{n}$ are constants satisfying $\alpha_{i}^{2}+\beta_{i}^{2} \neq 0$. From (5.4) we obtain

$$
\begin{aligned}
(\varphi \circ \gamma)_{1}=\sum_{k=0}^{\infty} & \sum_{i=1}^{N}\left\{(-1)^{k} \frac{(2 \pi r)^{2 k}}{(2 k) !} c_{i}\left(\alpha_{i} \cos \left(\frac{s}{r}\right)+\beta_{i} \sin \left(\frac{s}{r}\right)\right)^{2 k}\right. \\
& \left.+(-1)^{k} \frac{(2 \pi r)^{2 k+1}}{(2 k+1) !} d_{i}\left(\alpha_{i} \cos \left(\frac{s}{r}\right)+\beta_{i} \sin \left(\frac{s}{r}\right)\right)^{2 k+1}\right\} .
\end{aligned}
$$

Since $N$ is finite, the function

$$
u(s)=\sum_{k=0}^{\infty}(-1)^{k} \frac{(2 \pi r)^{2 k}}{(2 k) !} \sum_{i=1}^{N} c_{i}\left(\alpha_{i} \cos \left(\frac{s}{r}\right)+\beta_{i} \sin \left(\frac{s}{r}\right)\right)^{2 k}
$$


is not an algebraic polynomial in $\cos (s / r)$ and $\sin (s / r)$ unless $u(s)=0$ identically. Similarly,

$$
v(s)=\sum_{k=0}^{\infty}(-1)^{k} \frac{(2 \pi r)^{2 k+1}}{(2 k+1) !} \sum_{i=1}^{N} d_{i}\left(\alpha_{i} \cos \left(\frac{s}{r}\right)+\beta_{i} \sin \left(\frac{s}{r}\right)\right)^{2 k+1}
$$

is not an algebraic polynomial in $\cos (s / r)$ and $\sin (s / r)$ unless $v(s)=0$ identically. Because $(\varphi \circ \gamma)_{1}$ is assumed to be nonconstant, at least one of $u(s)$ and $v(s)$ is of infinite type, by Proposition 2.2. Thus, $\varphi \circ \gamma$ is of infinite type.

As an application of Theorems 3.2, 4.2 and 5.1, we have the following result.

THeOREM 5.2. Let $x: M \rightarrow \mathbb{E}^{m}$ be a finite type isometric immersion of a compact irreducible symmetric space. Then we have

(1) $x$ carries each circle in every compact rank one totally geodesic submanifold of $M$ into a curve of finite type,

(2) $x$ carries each circle of every maximal torus of $M$ into a curve of infinite type whenever $\operatorname{rank}(M) \geq 2$.

Proof. (1) Assume that $N$ is a compact rank one totally geodesic submanifold of $M$. Since every circle $\gamma$ in $N$ is a homogeneous curve in $N$ by [9] and the group $I(N)$ of isometries on $N$ is a subgroup of $I(M)$ by [5], $\gamma$ is a homogeneous curve in $M$. Hence, by Theorem 3.2, $x$ carries $\gamma$ into a curve of finite type.

If $x: M \rightarrow \mathbb{E}^{m}$ is a finite type isometric immersion of a compact irreducible symmetric space, then Theorem 4.2 implies that $x$ carries each maximal torus $T^{r}$ of $M$ into a submanifold of finite type. Hence, by Theorem 5.1, $x$ carries every circle of $T^{r}$ into a curve of infinite type.

6. Isometric immersions which send circles to finite type curves. Let $x: M \rightarrow \mathbb{E}^{m}$ be an isometric immersion of a compact Riemannian manifold. It is proved in [10] that if, for some $r>0, x$ carries every circle with radius $r$ in $M$ into a circle (i.e., a 1-type curve) in $\mathbb{E}^{m}$, then $M$ is a Riemannian sphere and $x$ is the first standard imbedding of the sphere.

TheOREM 6.1. (1) Let $x: M \rightarrow \mathbb{E}^{m}$ be a finite type isometric immersion of a compact irreducible symmetric space. Then $M$ is of rank one if and only if, for some $r>0$, $x$ carries each circle with radius $r$ in $M$ into a curve of finite type.

(2) If $x: M \rightarrow \mathbb{E}^{m}$ is an isometric immersion of a compact rank one symmetric space which carries circles in $M$ into curves of finite type, then $x$ is of finite type.

Proof. Statement (1) follows from Theorem 3.2, statement (2) of Theorem 5.2, and the fact that every circle in a compact rank one symmetric space is a homogeneous curve.

To prove statement (2), let us assume $x: M \rightarrow \mathbb{E}^{m}$ is an isometric immersion of a compact rank one symmetric space which carries circles into curves of finite type. We want to prove that $x$ is a finite type immersion. We divide our proof into three cases. 
Case 1. $M=S^{n}$. First, we prove the result for $n=2$. Without loss of generality, we may regard $S^{2}$ as the unit sphere in $\mathbb{E}^{3}$ defined by $u_{0}^{2}+u_{1}^{2}+u_{2}^{2}=1$.

In order to prove that $x: S^{2} \rightarrow \mathbb{E}^{m}$ is of finite type, it suffices to prove that every infinite type isometric immersion of $S^{2}$ carries some circles with radius $r<1$ in $S^{2}$ into some curves of infinite type. This can be done as follows.

Recall that the $i$-th nonzero eigenvalue of the Laplacian $\Delta$ on $S^{2}$ is given by $\lambda_{i}=i(i+1)$ with multiplicity $m_{i}=2 i+1$. Moreover, it is also known that the restriction to $S^{2}$ of a harmonic homogeneous polynomial of degree $i$ in $u_{0}, u_{1}, u_{2}$ is an eigenfunction with eigenvalue $\lambda_{i}$. Conversely, if $f$ is an eigenfunction on $S^{2}$ with eigenvalue $\lambda_{i}$, then there is a unique harmonic homogeneous polynomial $P$ of degree $i$ in $u_{0}, u_{1}, u_{2}$ such that $f$ is the restriction of $P$ to $S^{2}$ [2, pp. 159-162].

If $x=\left(x_{1}, \ldots, x_{m}\right)$ is an infinite type isometric immersion of $S^{2}$ into $\mathbb{E}^{m}$, then there is an $x_{A}$, where $A=1, \ldots, m$, whose spectral decomposition contains infinitely many nonzero terms. Hence, $x_{A}$ is an infinite series in $u_{0}, u_{1}, u_{2}$ that is not an algebraic polynomial in $u_{0}, u_{1}, u_{2}$. Put

$$
x_{A}=\sum a_{i_{0} i_{1} i_{2}} u_{0}^{i_{0}} u_{1}^{i_{1}} u_{2}^{i_{2}}
$$

Then at least one of $u_{0}, u_{1}, u_{2}$ has power which approaches to $\infty$. Without loss of generality, we may assume that the power of $u_{1}$ goes to infinity, i.e.

$$
\sup \left\{i_{1}: a_{i_{0} i_{1} i_{2}} \neq 0\right\}=\infty \text {. }
$$

Now, consider a circle $\gamma$ of $S^{2}$ of radius $r<1$ given by the intersection of $S^{2}$ with the plane defined by $u_{0}=\sqrt{1-r^{2}}$. We put $u_{1}=r \cos (s / r), u_{2}=r \sin (s / r)$. Substituting these equations into (6.1) yields

$$
x_{A}=\sum a_{i_{0} i_{1} i_{2}} r^{i_{1}+i_{2}} c^{i_{0}} \cos ^{i_{1}}\left(\frac{s}{r}\right) \sin ^{i_{2}}\left(\frac{s}{r}\right) .
$$

Since $\sup \left\{i_{1}: a_{i_{0} i_{1} i_{2}} \neq 0\right\}=\infty,(6.2)$ cannot be an algebraic polynomial in $\cos (s / r)$ and $\sin (s / r)$. Thus $x(\gamma)$ must be a curve of infinite type by Proposition 2.2. Hence, if the immersion of $S^{2}$ carries circles into curves of finite type, it is of finite type.

Next, assume $n>2$ and $x: S^{n} \rightarrow \mathbb{E}^{m}$ carries circles into curves of finite type. Then $x$ carries circles in a totally geodesic 2-sphere $S^{2}$ of $S^{n}$ into curves of finite type. Thus, by applying the result we have just proved, the restriction of $x$ to every totally geodesic $S^{2}$ is of finite type. Hence, by Lemma 4.1, $x$ carries geodesics in $S^{2}$ into curves of finite type. Because every geodesic in $S^{n}$ is contained in some totally geodesic $S^{2}$, it follows that $x$ carries each geodesic in $S^{n}$ into a curve of finite type. Hence, $x$ is of finite type by Lemma 4.1 .

Case 2. $M=R P^{n}(n \geq 2)$. Let $\pi: S^{n} \rightarrow R P^{n}$ be the projection from $S^{n}$ onto $R P^{n}$. Then the $i$-th eigenspace $V_{i}$ associated with the $i$-th nonzero eigenvalue of the Laplacian on $R P^{n}$ consists of those functions $f$ for which $f \circ \pi$ belongs to the $2 i$-th eigenspace of $S^{n}$.

If $x: R P^{2} \rightarrow \mathbb{E}^{m}$ is an isometric immersion of infinite type, then there is an $x_{A}$ whose spectral decomposition contains infinitely many nonzero terms. Hence, if we express $x_{A}$ in the form of (6.1), then at least one of the powers of $u_{0}, u_{1}, u_{2}$ must approach $\infty$. Hence, by applying the same argument as given in case (1) for $S^{2}$, we know that $x$ carries some circles in $R P^{2}$ into curves of infinite type. Consequently, if 
$x: R P^{2} \rightarrow \mathbb{E}^{m}$ carries every circle with radius $r$ into a curve of finite type, then $x$ must be of finite type. Therefore, $x$ must carry each geodesic in $R P^{2}$ into a curve of finite type, by Lemma 4.1 .

Next, let us assume that $n>2$ and $x: R P^{n} \rightarrow \mathbb{E}^{m}$ is an isometric immersion which carries every circle in $R P^{n}$ into a curve of finite type. Then $x$ carries every circle in a totally geodesic $R P^{2}$ of $R P^{n}$ into a curve of finite type. Hence, by applying the result we have just proved for $R P^{2}$, we conclude that the restriction of $x$ to the totally geodesic $R P^{2}$ is of finite type. Hence, it carries every geodesic in the totally geodesic $R P^{2}$ into a curve of finite type. Since every geodesic in $R P^{n}$ is contained in some totally geodesic $R P^{2}, x$ carries every geodesic in $R P^{n}$ into a curve of finite type. Consequently, by applying Lemma 4.1 again, we conclude that $x: R P^{n} \rightarrow \mathbb{E}^{m}$ is of finite type.

Case 3. $M=C P^{n}, H P^{n}$ or the Cayley plane $O P^{2}$. Let $\beta$ be a given geodesic in $M$. Then $\beta$ is contained in a totally geodesic $C P^{1}, H P^{1}$ or the Cayley line $\mathcal{O} P^{1}$ of $C P^{n}, H P^{n}$ or $\mathcal{O} P^{2}$, respectively. Recall that $C P^{1}, H P^{1}$ and $\mathcal{O} P^{1}$ are $k$-spheres $S^{k}$ with $k=2,4$ or 8 , respectively. Clearly, a circle with radius $r$ in $S^{k}$ is also a circle in $M$ with the same radius. Hence, if $x: M \rightarrow \mathbb{E}^{m}$ carries every circle with radius $r$ in $M$ into a curve of finite type, then the restriction of $x$ to $S^{k}$ must carry each circle with radius $r$ in $S^{k}$ into a curve of finite type. Therefore, by case (1), we know that $x: S^{k} \rightarrow \mathbb{E}^{m}$ is an immersion of finite type. Hence $x$ carries geodesic $\beta$ into a curve of finite type, by Lemma 4.1. Because $\beta$ can be chosen to be any geodesic in $M, x$ carries each geodesic in $M$ into a curve of finite type. Consequently, $x: M \rightarrow \mathbb{E}^{m}$ is of finite type by Lemma 4.1.

ACKNOWLEDGEMENTs. The author is grateful to the referee for several valuable suggestions.

\section{REFERENCES}

1. C. Baikoussis, F. Defever, T. Koufogiorgos and L. Verstraelen, Finite type immersions of flat tori into Euclidean spaces, Proc. Edinburgh Math. Soc. (2) 38 (1995), 413-420.

2. M. Berger, P. Gauduchon and $\mathrm{E}$. Mazet, Le spectre d'une variété riemannienne (Springer-Verlag, 1971).

3. B.-Y. Chen, Total mean curvature and submanifolds of finite type (World Scientific, 1984).

4. B.-Y. Chen, J. Deprez and P. Verheyen, Immersions, dans un espace euclidien, d'un espace symétrique compact de rang un à géodésiques simples, C. R. Acad. Sci. Paris, Sér. I Math. 304 (1987), 567-570.

5. B.-Y. Chen and T. Nagano, Totally geodesic submanifolds of symmetric spaces, Duke Math. J. 44 (1997), 117-129; B.-Y. Chen and T. Nagano, Totally geodesic submanifolds of symmetric spaces, II, Duke Math. J. 45 (1978), 405-425.

6. B.-Y. Chen and M. Petrovic, On spectral decomposition of immersions of finite type, Bull. Austral. Math. Soc. 44 (1991), 745-755.

7. J. Deprez, Immersions of finite type of compact homogeneous Riemannian manifolds, Doctoral Thesis (Katholieke Universiteit Leuven 1988).

8. S. Helgason, Differential geometry, Lie groups and symmetric spaces (Academic Press, 1978).

9. K. Mashimo and K. Tojo, Circles in Riemannian symmetric spaces, Kodai Math. J. 22 (1999), 1-14.

10. K. Nomizu and K. Yano, On circles and spheres in Riemannian geometry, Math. Ann. 210 (1974), 163-170.

11. B. O'Neill, Semi-Riemannian geometry (Academic Press, 1983).

12. L. Verstraelen, On submanifolds of finite Chen type and of restricted type, Results Math. 20 (1991), 744-755. 